\title{
Characteristics in Molecular Vibrational Frequency Patterns between Agonists and Antagonists of Histamine Receptors
}

\author{
S. June Oh \\ Department of Pharmacology, Inje University College of Medicine, Busan 614-735, Korea
}

http://dx.doi.org/10.5808/Gl.2012.10.2.128

Genomics Inform 2012;10(2):128-132

The legend of Figure 1 was given incorrectly. Histidine should be corrected as histamine. 